\title{
FATORES PROGNÓSTICOS CLÍNICOS E ANATOMOPATOLÓGICOS DOS TUMORES ESTROMAIS GASTROINTESTINAIS (GIST) DE ORIGEM GÁSTRICA
}

\author{
CLINICAL AND PATHOLOGICAL PROGNOSTIC FACTORS INVOLVED IN \\ GASTROINTESTINAL STROMAL TUMORS (GIST) OF GASTRIC ORIGIN
}

\author{
Marcus Valadão, TCBC-RJ'1 ; Laércio Gomes Lourenço, TCBC-SP²; Eduardo Linhares, TCBC-RJ³; \\ Sérgio Romano ${ }^{4}$ : Rubens Kesley ${ }^{5}$; Deborah Siqueira $^{6}$
}

\begin{abstract}
RESUMO: Objetivo: Identificar os fatores prognósticos clínicos e anatomopatológicos nos portadores de tumor estromal gastrointestinal (GIST) gástrico submetidos à ressecção cirúrgica. Método: Estudo retrospectivo realizado no Instituto Nacional do Câncer (INCA), incluindo 29 casos de GIST gástrico c-Kit positivo submetidos à ressecção cirúrgica entre 1983 e 2004. Variáveis clínicas e anatomopatológicas foram investigadas quanto ao significado prognóstico, correlacionando-as com sobrevida global e sobrevida livre de doença. Resultados: O acompanhamento mediano foi de 35 meses. A sobrevida global, estimada em cinco anos, foi de 53\%. As variáveis tamanho tumoral maior que $13,5 \mathrm{~cm}$ e presença de recidiva tiveram implicação prognóstica na sobrevida global conforme análise univariada ( $\mathrm{p}=0,01 \mathrm{e} \mathrm{p}=0,03$, respectivamente). A análise multivariada evidenciou que tamanho tumoral maior que $13,5 \mathrm{~cm}$ e presença de recidiva representaram fatores prognósticos independentes relacionados à sobrevida global $(\mathrm{p}=0,01 \mathrm{e} p=0,03$, respectivamente). As características tamanho tumoral maior que $13,5 \mathrm{~cm}$ e índice mitótico influenciaram significativamente $(\mathrm{p}=0,04 \mathrm{e} \mathrm{p}=0,04)$ a sobrevida livre de doença (análise univariada), porém apenas tamanho tumoral maior que $13,5 \mathrm{~cm}$ apresentou-se como fator prognóstico independente $(\mathrm{p}=0,04)$ relacionado à sobrevida livre de doença, quando utilizada a análise multivariada. Conclusão: $\mathrm{O}$ tamanho tumoral e a presença de recidiva representaram fatores prognósticos independentes relacionados à sobrevida global. Apenas o tamanho tumoral apresentou impacto adverso independente na sobrevida livre de doença (Rev. Col. Bras. Cir. 2006; 33(5): 298-304).
\end{abstract}

Descritores: Sarcoma; Neoplasias gástricas; Prognóstico; Sobrevivência.

\section{INTRODUÇÃO}

O conhecimento gerado nos últimos anos acerca dos mecanismos moleculares envolvidos na patogênese do GIST foi responsável por sua identificação como entidade clínica distinta e pela compreensão de seu comportamento biológico. O melhor conhecimento sobre sua história natural permitiu observar que a cirurgia representa a única modalidade terapêutica capaz de conferir cura, porém, utilizada isoladamente, apresenta resultados desanimadores, visto que apenas $30 \%$ a $40 \%$ dos pacientes atingem critérios de cura após longo acompanhamento ${ }^{1}$. Por outro lado, o desenvolvimento do mesilato de imatinibe trouxe benefícios para os casos de doença metastática e irressecável ${ }^{2-4}$, produzindo resultados animadores e trazendo à tona novas perspectivas de utilização como tratamento adjuvante/neoadjuvante ${ }^{5,6}$, com o intuito de otimizar os resultados da cirurgia, aumentando as chances de cura para os portadores desta neoplasia. Baseado nesses fatos, a identificação dos fatores prognósticos responsáveis pelos resultados insatisfatórios torna-se assunto de funda- mental importância, pois essas informações proporcionarão a estratificação de grupos de pior prognóstico (alto risco para recidiva ou menor sobrevida), permitindo que esse grupo se torne base para estudos futuros que investiguem os benefícios de terapias adjuvantes pós-ressecções cirúrgicas.

O estudo objetivou identificar fatores prognósticos clínicos e anatomopatológicos em portadores de tumor estromal gastrointestinal (GIST) gástrico submetidos à ressecção cirúrgica, correlacionando-os com a sobrevida global e a sobrevida livre de doença.

\section{MÉTODO}

Estudo retrospectivo realizado no Instituto Nacional do Câncer (INCA-RJ) através da identificação (Serviço de Cirurgia Abdômino-Pélvica) de 41 doentes com diagnóstico sugestivo de GIST submetidos à ressecção cirúrgica entre 1983 e 2004. Todas as lâminas foram revisadas pelos mesmos patologistas (Serviço de Anatomia-Patológica do INCA), sendo realizada pesquisa da proteína c-Kit (CD117) por imuno-

1. Cirurgião do INCA.

2. Professor Adjunto da Escola Paulista de Medicina-Universidade Federal de São Paulo.

3. Cirurgião da Seção de Cirurgia Abdômino-Pélvica do INCA; Mestre e Doutor em Cirurgia pela UFRJ.

4. Patologista do Serviço de Anatomia Patológica do INCA.

5. Cirurgião do INCA; Professor da Universidade Gama Filho; Mestre em Cirurgia.

6. Ex-Residente do Serviço de Anatomia Patológica do INCA; Patologista do Serviço de Anatomia Patológica do Hospital dos Servidores do EstadoRJ.

Recebido em 25-05-2006

Aceito para publicação em 30-06-2006

Conflito de interesses: nenhum

Fonte de financiamento: nenhuma

Trabalho realizado na Seção de Cirurgia Abdômino-Pélvica do Instituto Nacional do Câncer (INCA). Parte da Tese apresentada à Universidade Federal de São Paulo- Escola Paulista de Medicina-Programa de Pós-Graduação em Gastroenterologia Cirúrgica, para obtenção do Título de Mestre em Ciências. 
histoquimica em todos os casos. Os doentes foram alocados no estudo respeitando-se os seguintes critérios de inclusão: portadores de GIST gástrico com confirmação imunohistoquímica (CD117 positivo) submetidos à ressecção cirúrgica macroscopicamente completa (R0 ou R1), operados na mesma instituição (Instituto Nacional do Câncer-INCA) no período compreendido entre 1983 e 2004. A grande maioria dos casos reconhecidos como GIST após o estudo imunohistoquímico tinham tido o diagnóstico de outros tipos histológicos como leiomioma, leiomiossarcoma e leiomioblastoma.

Foram excluídos 12 casos, utilizando-se dos seguintes critérios: realização de ressecção cirúrgica R2 (doença residual macroscópica) (um caso); GIST c-Kit negativo (dois casos); GIST de localização não-gástrica (quatro casos); informações incompletas relativas ao tumor primário (tumor primário operado em outras instituições) ( cinco casos).

Foram analisadas variáveis clínicas e anatomopatolológicas relacionadas ao GIST, correlacionandoas com sobrevida global e sobrevida livre de doença. A análise estatística foi realizada através do programa SPSS versão 8.0. A sobrevida foi estimada pelo método de Kaplan-Meier e as diferenças estatísticas entre os grupos foram analisadas pelo Teste de Log-rank. Os fatores prognósticos relacionados à sobrevida global e à sobrevida livre de doença identificados na análise univariada foram submetidos à análise multivariada através do Teste de Regressão de Cox. O nível de significância estatística utilizada foi $\mathrm{p}<0,05$.

\section{RESULTADOS}

Os dados referentes às características da amostra $(n=29)$ estão explicitadas na Tabela 1 . A idade mediana foi de 63 anos, variando de 10 a 81 anos.

Foi realizada gastrectomia total em nove casos (31\%), gastrectomia subtotal em sete casos $(24,1 \%)$ e gastrectomia segmentar em 13 pacientes (44,9\%). Houve necessidade de ressecção poliorgânica em 13 casos. Dois pacientes apresen-

Tabela 1 - Características dos pacientes $(n=29)$.

\begin{tabular}{lcc}
\hline Características & \multicolumn{1}{c}{ Número de pacientes (percentual) } \\
\hline Sexo & 10 & $(34,4 \%)$ \\
$\quad$ Masculino & 19 & $(65,6 \%)$ \\
$\quad$ Feminino & & \\
Dor & 19 & $(65,6 \%)$ \\
$\quad$ Presente & 10 & $(34,4 \%)$ \\
$\quad$ Ausente & & \\
Massa Palpável & 16 & $(55,1 \%)$ \\
$\quad$ Presente & 13 & $(44,9 \%)$ \\
$\quad$ Ausente & & \\
Perda De Peso & 12 & $(41,3 \%)$ \\
$\quad \begin{array}{l}\text { Presente } \\
\text { Ausente }\end{array}$ & 17 & $(58,7 \%)$ \\
Hemorragia Digestiva & & \\
$\quad$ Presente & 12 & $(41,3 \%)$ \\
$\quad$ Ausente & 17 & $(58,7 \%)$ \\
\hline$\quad$ & &
\end{tabular}

taram rotura tumoral durante o procedimento cirúrgico e outro caso apresentou hemoperitôneo devido a rotura tumoral espontânea. Onze doentes (37,9\%) necessitaram hemotransfusão no per-operatório. A média de linfonodos ressecados por procedimento foi de 10 (a linfadenectomia não foi empregada de rotina).

A Tabela 2 resume as características anatomopatológicas da amostra estudada.

O acompanhamento mediano foi de 35 meses (variou de 6 a 134). No ponto utilizado como censo, 19 doentes estavam vivos, sendo que 11 permaneciam sem evidência de doença. A sobrevida global em cinco anos foi de 53\%. A taxa de recorrência global foi de $58,6 \%(n=17)$. Os portadores de recidiva cursaram com sobrevida significativamente menor que o grupo sem recidiva $(p=0,03)$. Dos doentes que apresentaram recidiva $(n=17)$, cinco utilizaram mesilato de imatinibe, sendo que quatro casos ainda não haviam completado um ano de tratamento com a medicação. Não houve diferença estatisticamente significante na sobrevida com a utilização do mesilato de imatinibe $(p=0,13)$.

Dos fatores testados na análise univariada, aqueles que tiveram impacto adverso estatisticamente significante na sobrevida foram: presença de recidiva $(p=0,03)$, tamanho tumoral maior que $13,5 \mathrm{~cm}(p=0,01)$ e necessidade de ressecção de órgãos associados $(p=0,02)$ (Tabela 3$)$. O estudo da variável ressecção poliorgânica estratificada pelo tamanho tumoral (mediana) evidenciou não haver implicação prognóstica dessa variável na sobrevida global (Log-Rank 3,78; Grau de Liber$\operatorname{dade}(\mathrm{Glib})=1 ; \mathrm{p}=0,05)$.

As curvas de sobrevida relacionadas as variáveis estatisticamente significantes estão explicitadas na Figura 1.

As características identificadas na análise univariada foram submetidas à análise multivariada, sendo evidenciado que as variáveis tamanho tumoral (maior que $13,5 \mathrm{~cm}$ ) e a presença de recidiva representaram fatores prognósticos independentes relacionado à sobrevida (Tabela 4).

Das variáveis estudadas, aquelas que tiveram associação estatisticamente significante com o desenvolvimento

Tabela 2 - Características anatomopatológicas.

\begin{tabular}{lrl}
\hline Características & \multicolumn{2}{c}{ Número de pacientes (percentual) } \\
\hline Índice Mitótico & & \\
$\quad>5$ Mitoses/50 Cga* & 19 & $(65,6 \%)$ \\
$\quad<5$ Mitoses/50 Cga & 10 & $(34,4 \%)$ \\
Localização & & \\
$\quad$ Proximal & 20 & $(69 \%)$ \\
$\quad$ Distal & 9 & $(31 \%)$ \\
Tipo Histológico & & \\
$\quad$ Fusiforme & 15 & $(51,8 \%)$ \\
$\quad$ Epitelióide & 3 & $(10,3 \%)$ \\
$\quad$ Misto & 11 & $(37,9 \%)$ \\
Metástase Linfonodal & & \\
$\quad$ Presente & 2 & $(6,8 \%)$ \\
$\quad$ Ausente & 27 & $(93,2 \%)$ \\
\hline
\end{tabular}

*CGA = Campo de Grande Aumento. 
de recidiva tumoral (análise univariada) foram: tamanho tumoral maior que $13,5 \mathrm{~cm}(\mathrm{p}=0,04)$ e índice mitótico elevado $(>5$ mitoses/ 50 CGA) $(p=0,04)$ (Tabela 5).

As curvas de sobrevida livre de doença dos fatores implicados na recidiva $(\mathrm{p}<0,05)$ estão representadas na Figura 2.

Os fatores prognósticos associados à presença de recidiva evidenciados pela análise univariada foram submeti-

Tabela 3 - Fatores prognósticos relacionados à sobrevida global (análise univariada).

\begin{tabular}{|c|c|c|c|}
\hline Variável & Log-Rank & Glib & $\mathbf{p}$ \\
\hline Idade & 1,08 & 1 & 0,29 \\
\hline Sexo & 0,90 & 1 & 0,34 \\
\hline Dor & 0,37 & 1 & 0,54 \\
\hline Massa Palpável & 2,84 & 1 & 0,09 \\
\hline Hemorragia digestiva & 0,0 & 1 & 0,99 \\
\hline Perda de peso & 1,06 & 1 & 0,30 \\
\hline Hemotransfusão & 0,31 & 1 & 0,57 \\
\hline Tipo de operação & 1,06 & 2 & 0,28 \\
\hline Tamanho $(5 \mathrm{~cm})$ & 0,17 & 1 & 0,68 \\
\hline Tamanho $(10 \mathrm{~cm})$ & 0,22 & 1 & 0,64 \\
\hline Metástase linfonodal & 0,20 & 1 & 0,65 \\
\hline Índice mitótico & 3,0 & 1 & 0,05 \\
\hline Localização & 0,20 & 1 & 0,65 \\
\hline Tipo histológico & 1,68 & 2 & 0,43 \\
\hline Presença de metástase & 0,96 & 1 & 0,32 \\
\hline Rotura tumoral & 3,16 & 1 & 0,07 \\
\hline CD34 & 1,08 & 1 & 0,29 \\
\hline Vimentina & 0,35 & 1 & 0,55 \\
\hline ENS & 1,58 & 1 & 0,20 \\
\hline $\mathrm{S} 100$ & 1,50 & 1 & 0,25 \\
\hline Desmina & 1,07 & 1 & 0,30 \\
\hline HHF35 & 1,00 & 1 & 0,31 \\
\hline Mesilato de imatinibe & 2,24 & 1 & 0,13 \\
\hline Invasão de órgãos & 3,8 & 1 & 0,05 \\
\hline Tamanho $>13,5 \mathrm{~cm}$ & 5,75 & 1 & 0,01 \\
\hline Ressecção poliorgânica & 5,03 & 1 & 0,02 \\
\hline Recidiva & 4,46 & 1 & 0,03 \\
\hline
\end{tabular}

Recidiva X Sobrevida

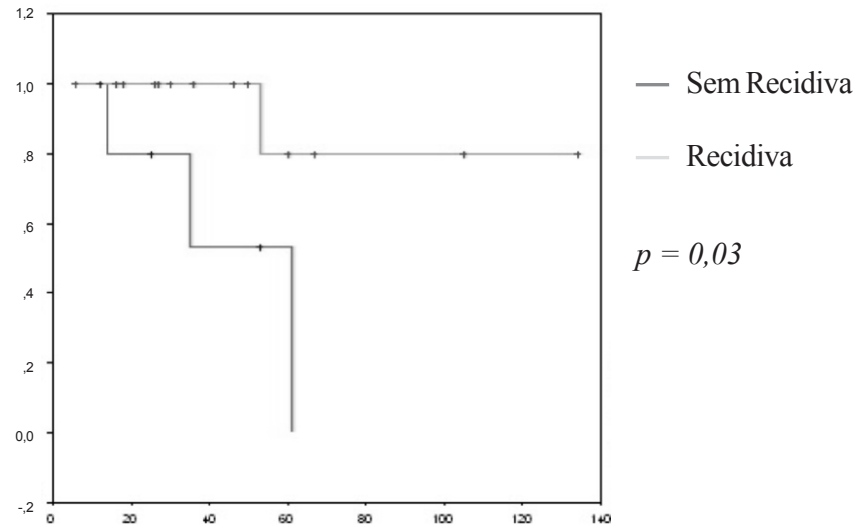

Tabela 4 - Resultado da análise multivariada dos fatores que tiveram impacto na sobrevida $(p<0,05)$ pela análise univariada.

\begin{tabular}{lccc}
\hline Variáveis & Hazard Ratio & IC 95\% & P \\
\hline Recidiva & 4,4 & $0,1-28,9$ & 0,03 \\
Tamanho Tumoral & 5,7 & $0,08-10,0$ & 0,01 \\
\hline
\end{tabular}

Likelihood Ratio Teste Qui Quadrado:38,20, Glib=1, $(p<0,0062>)$. IC: Intervalo de confiança.

dos à análise multivariada, sendo identificado tamanho tumoral (maior que 13,5 cm) como fator prognóstico independente relacionado ao desenvolvimento da recorrência tumoral $(\mathrm{p}=0,04$; IC 95\%=0,7-55,3; Hazard ratio=3,9: Likelihood Ratio Teste Qui Quadrado:28,99, Glib=1, $<<0,04>$ ).

\section{DISCUSSÃO}

A comunidade científica tem buscado identificar, entre as inúmeras variáveis estudadas, aquelas que possam ser capazes de predizer, de modo confiável, a evolução dos portadores de GIST. Nesse sentido, têm-se realizado uma grande quantidade de estudos com a finalidade de identificar fatores prognósticos, porém os resultados obtidos apresentam enorme diversidade. Isto se deve, em parte, pela constatação de que a maioria desses estudos apresenta análise distorcida dos seus dados por incluírem grupos heterogêneos de casos, além de apresentarem casuísticas pouco expressivas.

Essas observações contribuíram para que fosse definida, de modo criterioso, a amostra a ser estudada no presente trabalho, a fim de se obter uma casuística homogênea e evitar viéses. Assim sendo, a análise restringiu-se aos portadores de GIST de origem gástrica, tendo como base evidências de que a localização do tumor primário teria influência na sobrevida $^{7}$ e que os fatores prognósticos pesquisados teriam características peculiares dependendo do sítio de origem do tumor. Um desses exemplos é que o tamanho tumoral utilizado como fator prognóstico no GIST gástrico seria de $10 \mathrm{~cm}$ e não $5 \mathrm{~cm}$ como é utilizado para o GIST proveniente do intestino delgado ${ }^{7}$. Além disso, foram excluídos todos os GIST c-Kit

Tamanho Tumoral X Sobrevida

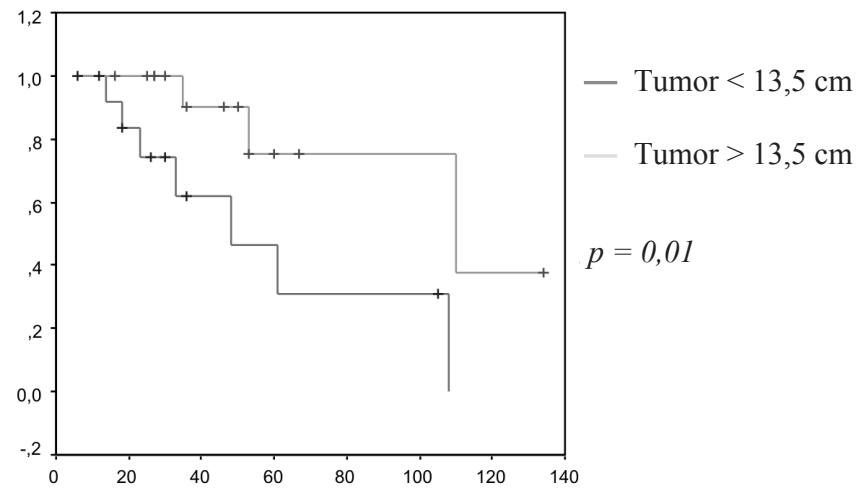

Figura 1 - Curvas de sobrevida relacionadas à recidiva e ao tamanho tumoral. 
negativo, mantendo-se a premissa da homogeneidade da amostra. Três casos submetidos à cirurgia R1 (doença microscópica residual) foram incluídos para análise (dois por rotura tumoral durante o procedimento cirúrgico e um devido a hemoperitôneo por rotura tumoral espontânea) por haver evidência na literatura de que essas variáveis têm influência na sobrevida ${ }^{8,9}$.

Tabela 5 - Análise univariada dos fatores implicados no desenvolvimento de recidiva tumoral (sobrevida livre de doença).

\begin{tabular}{lccc}
\hline Variável & Log-Rank & $\begin{array}{c}\text { Grau de } \\
\text { liberdade }\end{array}$ & p \\
\hline Idade & 0,01 & 1 & 0,90 \\
Sexo & 0,10 & 1 & 0,75 \\
Dor & 0,66 & 1 & 0,41 \\
Massa Palpável & 0,04 & 1 & 0,84 \\
Hemorragia digestiva & 1,18 & 1 & 0,27 \\
Perda de peso & 0,22 & 1 & 0,63 \\
Hemotransfusão & 0,64 & 1 & 0,42 \\
Tipo de operação & 1,60 & 2 & 0,44 \\
Tamanho (5cm) & 0,48 & 1 & 0,48 \\
Tamanho (10cm) & 0,19 & 1 & 0,66 \\
Metástase linfonodal & 0,31 & 1 & 0,57 \\
Localização & 1,40 & 1 & 0,23 \\
Tipo histológico & 0,45 & 2 & 0,79 \\
Presença de metástase & 0,65 & 1 & 0,41 \\
Rotura tumoral & 1,50 & 1 & 0,20 \\
CD34 & 1,24 & 1 & 0,26 \\
Vimentina & 0,29 & 1 & 0,58 \\
ENS & 0,33 & 1 & 0,56 \\
S100 & 2,0 & 1 & 0,09 \\
Desmina & 1,32 & 1 & 0,25 \\
HHF35 & 1,30 & 1 & 0,28 \\
Ressecção poliorgânica & 2,19 & 1 & 0,13 \\
Invasão de órgãos & 1,12 & 1 & 0,29 \\
Índice mitótico & 3,70 & 1 & 0,04 \\
Tamanho>13,5 cm & 3,70 & 1 & 0,04 \\
\hline
\end{tabular}

Índice Mitótico X Sobrevida Livre de Doença

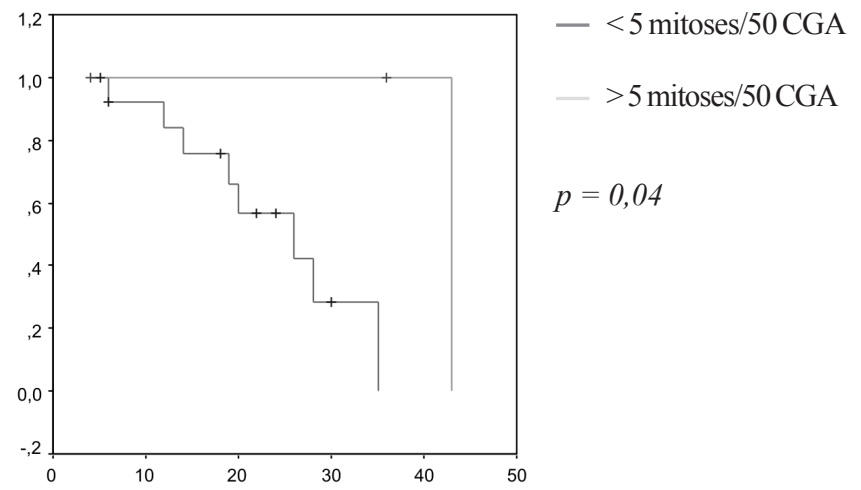

Em relação aos resultados encontrados, pode-se observar que houve predominância do sexo feminino $(65,6 \%)$, fato que contraria os achados da literatura ${ }^{10}$, que não evidencia diferença na incidência entre os sexos. A idade mediana ao diagnóstico (63 anos) foi comparável aos dados da literatura ${ }^{1}$, inclusive a incidência baixa de tumores nos extremos de idade (apenas dois casos abaixo de 40 anos e três acima dos 75 anos).

A maioria dos doentes apresentava sintomas como dor abdominal, perda de peso, massa palpável e hemorragia digestiva, havendo apenas dois assintomáticos. Essa observação pode ser explicada pelo fato de que a grande maioria da amostra estudada apresentava lesões volumosas (tamanho mediano de 13,5 cm; apenas dois doentes apresentavam tumores menores que $5 \mathrm{~cm}$ ), o que contribui para o desenvolvimento da sintomatologia.

Apesar da maioria dos tumores se localizarem na porção proximal do estômago (69\%), apenas 31\% dos casos foram submetidos à gastrectomia total, sendo a maior parte dos procedimentos $(44,9 \%)$ representada pela gastrectomia segmentar. Esse dado denota a factibilidade da preservação do estômago no tratamento do GIST gástrico ${ }^{11}$, sendo possível a ressecção conservadora, desde que seja assegurada margens livres de 1 a $2 \mathrm{~cm}$ checadas por estudo histopatológico de congelação no intuito de reduzir a morbidade do procedimento e proporcionar melhor qualidade de vida.

Houve necessidade de ressecção poliorgânica em 13 pacientes $(44,9 \%)$, porém em apenas sete deles havia confirmação microscópica de invasão. Esses achados podem ser explicados pela grande proporção de lesões volumosas (tamanho mediano de $13,5 \mathrm{~cm}$ ) despertando a possibilidade de invasão de órgãos vizinhos e a necessidade de ressecção em bloco para se obter margens livres.

A sobrevida global (53\% em cinco anos) e a taxa de recorrência $(58,6 \%)$ foram comparáveis aos resultados das maiores séries publicadas na literatura ${ }^{1,6-8}$, confirmando os resultados insatisfatórios com a utilização da cirurgia como terapêutica isolada.

Das variáveis testadas, identificou-se o tamanho tumoral maior que $13,5 \mathrm{~cm}$ e a presença de recidiva como fatores prognósticos independentes relacionados à sobrevida glo-

Tamanho Tumoral X Sobrevida Livre de Doença

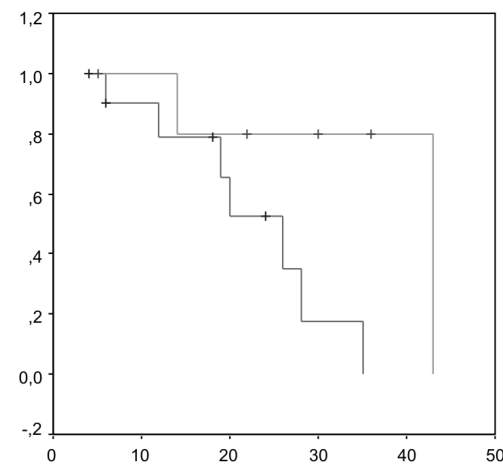

Figura 2 - Curvas de sobrevida livre de doença relacionadas a tamanho tumoral (maior que 13,5cm) e índice mitótico. 
bal (análise multivariada). Quanto à sobrevida livre de doença, apenas o tamanho tumoral maior que $13,5 \mathrm{~cm}$ representou influência prognóstica pela análise multivariada.

Esses dados confirmam os achados evidenciados na literatura ${ }^{1,4,8,12}$, identificando o tamanho tumoral e a presença de recidiva como variáveis prognósticas independentes. $\mathrm{O}$ tamanho tumoral tem sido reconhecido como fator adverso na grande maioria das publicações sobre GIST, porém o diâmetro a ser considerado como divisor de águas no GIST gástrico é motivo de debate. Emory et al. ${ }^{7}$ evidenciaram que tumores de tamanho semelhantes apresentavam melhor sobrevida quando tinham origem gástrica quando comparado aos originados do intestino delgado, evidenciando que o ponto de corte de $10 \mathrm{~cm}$ pudesse ser o mais indicado em predizer o comportamento do GIST gástrico. O presente estudo evidenciou que a utilização do ponto de corte de 13,5 $\mathrm{cm}$ (mediana de tamanho da amostra estudada) influenciou na sobrevida global e na sobrevida livre de doença, fato que não aconteceu quando foram utilizadas as referências de $5 \mathrm{~cm}$ e $10 \mathrm{~cm}$. Este resultado reforça a observação de que a utilização do parâmetro $5 \mathrm{~cm}$ de diâmetro não parece ser o mais adequado na predição do comportamento biológico dos GIST de origem gástrica. $\mathrm{O}$ achado de $13,5 \mathrm{~cm}$ e não $10 \mathrm{~cm}$ como fator prognóstico pode estar relacionado às características da amostra (apenas 29 casos, sendo a maioria representada por tumores de grande diâmetro).

A presença de recidiva tem sido implicada como variável preditora de pior sobrevida ${ }^{12,13}$. Mudan et al. ${ }^{12}$ evidenciaram que os portadores de recidiva cursavam com mediana de sobrevida de 15 meses, fato confirmado por Graadt et al. ${ }^{13}$. O resultado do presente trabalho corrobora essa observação, demonstrando que a presença de recidiva representou fator prognóstico independente relacionado a sobrevida.

A realização de ressecção poliorgânica não representou fator prognóstico relacionado à sobrevida global quando se utilizou a análise estratificada pelo tamanho tumoral. Pode-se entender que a ressecção de órgãos associados indica a presença de doença avançada, ou seja, associação de grande volume tumoral com a possibilidade de invasão de estruturas vizinhas. Embora a variável invasão de órgãos adjacentes não tenha tido significância estatística $(p=0,05)$, podese interpretar que o tamanho tumoral tenha tido influência mais importante como indicador da necessidade de ressecções poliorgânicas, tendo funcionado como fator confundidor em tal situação, fato comprovado pela análise estatística empregando-se estratificação pelo tamanho tumoral.

O índice mitótico tem sido reconhecido como um dos fatores prognósticos mais relevantes, sendo identificado na maioria das publicações ${ }^{14-18}$. Apesar do presente estudo não demonstrar correlação entre índice mitótico e sobrevida global, esta variável apresentou-se como fator prognóstico relacionado a sobrevida livre de doença pela análise univariada, porém não representou fator prognóstico independente na análise multivariada.

Os resultados obtidos no presente estudo corroboram os achados da literatura ${ }^{10,15,17,18}$ referente às variáveis não implicadas na sobrevida, como por exemplo: idade, sexo, características dos sintomas, tipo de cirurgia empregada e pre- sença de determinados marcadores imuno-histoquímicos. Quanto à idade, apenas uma publicação ${ }^{7}$ identificou-a como fator prognóstico, não sendo repetido em outros estudos. Quanto ao sexo, não há indícios de que essa variável tenha influência na evolução do GIST. Em relação às características dos sintomas, não há descrição na literatura da investigação dessa variável como fator preditor de comportamento biológico. Apesar de se imaginar que a presença de massa palpável e de hemorragia digestiva funcionassem indiretamente como fatores adversos, ambas não tiveram impacto na sobrevida global nem na sobrevida livre de doença.

Em relação ao tipo de operação empregada, os dados obtidos no presente trabalho confirmam os resultados da literatura ${ }^{8,15,17,18}$, evidenciando que a ressecção conservadora do estômago (ressecção segmentar) não influiu adversamente na sobrevida global e na sobrevida livre de doença quando comparada à realização de gastrectomia total ou subtotal. Esse achado reforça a idéia de que, quando possível, a ressecção segmentar do estômago deve ser utilizada no tratamento do GIST gástrico apesar de se imaginar que a presença de massa palpável, pela grande extensão tumoral, e da hemorragia digestiva, pela invasão extensa da mucosa além da necrose tumoral, sejam indiretamente, fatores adversos não tiveram impacto na sobrevida (assegurada margens negativas) pois, além de obter resultado oncológico eficaz, beneficiará o paciente no que tange à qualidade de vida ${ }^{19}$.

Dos marcadores imuno-histoquímicos empregados no presente estudo (CD34, vimentina, enolase neuro-específica, desmina e proteína S-100), nenhum teve influência na sobrevida, fato exaustivamente repetido na literatura9,10,16,17,18,20. Desta forma, ratifica-se a observação de que esses marcadores devem ser utilizados como auxiliares no diagnóstico de GIST e não como preditores prognósticos.

Foram observados resultados divergentes aos da literatura no que se refere ao estudo das seguintes variáveis: localização do tumor no estômago, tipo histológico, rotura tumoral, presença de metástase e utilização do mesilato de imatinibe. A localização do tumor no estômago e o tipo histológico não têm sido identificadas como variáveis prognósticas relacionadas ao GIST gástrico ${ }^{8,15,17,18}$, observação confirmada pelo presente estudo. Porém, Miettinen et al. ${ }^{10}$, analisando 1765 casos de GIST gástrico, demonstraram que os tumores localizados na região proximal do estômago tiveram pior prognóstico que os tumores distais. Além disso, esses autores subdividiram as características histológicas em oito grupos e evidenciaram que a variante sarcomatosa dos tipos fusiforme e epitelióide tiveram pior evolução. Apesar deste estudo representar a maior casuística de GIST gástrico publicada (1765 casos), não há relato na literatura a respeito desses achados. Desta forma, continua em aberto o papel dessas variáveis em predizer a evolução dos GIST gástricos.

Em relação à rotura tumoral, há evidências claras de que sua presença está relacionada a pior sobrevida ${ }^{8,9,21}$, porém essa observação não foi confirmada pelo presente trabalho, provavelmente devido à pequena amostra estudada (29 pacientes) e, conseqüentemente, o limitado poder para detectar diferença estatisticamente significante. 
A presença de metástase tem sido implicada como fator responsável por menor sobrevida ${ }^{1,13,15}$, porém a existência de metástase linfonodal não tem sido identificada como variável adversa ${ }^{1,21}$. A observação de que a presença de metástase não significou pior sobrevida pode ser explicada por alguns dados da amostra estudada: dois pacientes apresentavam metástase linfonodal enquanto três outros apresentavam metástases à distância, sendo todas as metástases ressecadas completamente (cirurgia R0), ou seja, a presença de metástase linfonodal associada à ressecção completa das metástases pode ter contribuído para que não fosse detectada diferença estatisticamente significante na sobrevida.

Apesar da evidência inequívoca na literatura de que a utilização do mesilato de imatinibe está relacionada ao aumento da sobrevida ${ }^{2-4,22}$, o resultado encontrado na presente amostra não confirma esse dado. Esse fato pode ser explicado pela observação de que, dos cinco doentes que utilizaram a medicação, apenas um havia completado um ano de tratamento farmacológico, denotando que o tempo curto de utilização ainda não seria suficiente para propiciar aumento de sobrevida, observação reforçada por evidências na literatura de que a sobrevida mediana dos portadores de recidiva varia entre $9 \mathrm{e}$ 15 meses sem a utilização do mesilato de imatinibe ${ }^{12,13}$.

Embora a hemotransfusão tenha sido implicada como variável prognóstica na oncologia ${ }^{23}$, sua pesquisa específica em relação ao GIST não tem sido relatada. Desta forma, o resultado encontrado (sem evidência de implicação prognóstica) deve aguardar confirmação de outros estudos.

Diante do exposto, fica evidente que a identificação de fatores prognósticos relacionados ao GIST é de fundamental importância, pois permite o melhor entendimento sobre a evolução natural da doença e a utilização dessas informações para adoção de novas estratégias terapêuticas. Assim, os resultados do presente estudo poderão servir de alicerce para pesquisas que investiguem a associação de tratamentos em portadores de GIST, visto que se pode identificar fatores prognósticos responsáveis por pior evolução e utilizá-los para se definir o grupo de pacientes que poderão se beneficiar desses tratamentos.

\begin{abstract}
Background: This study wants to identify clinical and pathologic prognostic factors of resected gastric gastrointestinal stromal tumors (GIST). Methods: Twenty-nine patients with c-Kit positive gastric GIST who underwent surgical resection at the Brazilian National Cancer Institute (INCA) between 1983 and 2004 were reviewed retrospectively. Prognostic significance of clinical and pathological variables was investigated. The endpoints were overall survival and disease free survival. Results: Median follow-up was 35 months. Five-year estimate survival rate was 53\%. Univariate analysis for overall survival identified size $>13.5 \mathrm{~cm}(p=0.01)$ and recurrence $(p=0.03)$ as prognostic factors. Size $>13.5 \mathrm{~cm}$ and recurrence were independent factors ( $p=0.01$ and $p=0.03$ respectively) in multivariate analysis. Univariate analysis for disease free survival identified size $>13.5 \mathrm{~cm}(p=0.04)$ and grade $(p=0.04)$ as prognostic factors but only size $>13.5 \mathrm{~cm}$ was an independent factor in multivariate analysis. Conclusion: Size $>13.5 \mathrm{~cm}$ and recurrence were identified as independent prognostic factors for overall survival. Only size $>13.5 \mathrm{~cm}$ was an independent prognostic factor for disease free survival.
\end{abstract}

Key words: Sarcoma; Stomach neoplasms; Prognosis; Survival

\section{REFERÊNCIAS}

1. DeMatteo RP, Lewis JJ, Leung D, Mudan SS, Woodruff JM, Brennan MF. Two hundred gastrointestinal stromal tumors: recurrence patterns and prognostic factors for survival. Ann Surg. 2000;231(1):51-8.

2. van Oosterom AT, Judson I, Verweij J, Stroobants S, di Paola ED, Dimitrijevic S, et al. Safety and efficacy of imatinib (STI571) in metastatic gastrointestinal stromal tumors: a phase I study. Lancet. 2001; 358(9291):1421-3.

3. Demetri GD, von Mehren M, Blanke CD, Van den Abbeele AD, Eisenberg B, Roberts PJ, et al. Efficacy and safety of imatinib mesylate in advanced gastrointestinal stromal tumors. N Engl J Med. 2002;347(7):472-80.

4. Verweij J, Casali PG, Zalcberg J, LeCesne A, Reichardt P, Blay JY, et al. Progression-free survival in gastrointestinal stromal tumours with high-dose imatinib: randomised trial. Lancet. 2004;364(9440):1127-34.

5. Valadão M, Linhares E, Mali Jr J, Pacheco JS, Stoduto G. Novas perspectivas no tratamento do GIST. Rev Bras Cancerol. No prelo 2006.

6. Eisenberg BL, Judson I. Surgery and imatinib in the management of GIST: emerging approaches to adjuvant and neoadjuvant therapy. Ann Surg Oncol. 2004;11(5):465-75.
7. Emory TS, Sobin LH, Lukes L, Lee DH, O’Leary TJ. Prognosis of gastrointestinal smooth-muscle (stromal) tumors: dependence on anatomic site. Am J Surg Pathol. 1999;23(1):82-7.

8. Ng EH, Pollock RE, Munsell MF, Atkinson EN, Romsdahl MM. Prognostic factors influencing survival in gastrointestinal leiomyosarcomas. Implications for surgical management and staging. Ann Surg. 1992;215(1):68-77.

9. Langer C, Gunawan B, Schüler P, Huber W, Füzesi L, Becker H. Prognostic factors influencing surgical management and outcome of gastrointestinal stromal tumours. Br J Surg. 2003;90(3):332-9.

10. Miettinen M, Sobin LH, Lasota J. Gastrointestinal stromal tumors of the stomach: a clinicopathologic, immunohistochemical, and molecular genetic study of 1765 cases with long term followup. Am J Surg Pathol. 2005;29(1):52-68.

11. Valadão M, Linhares E, Castro L, Pinto CE, Lugão R, Quadros $\mathrm{C}$, et al. GIST gástrico-Experiência do INCA. Rev Bras Cancerol. 2004;50(2):121-6.

12. Mudan SS, Conlon KC, Woodruff JM, Lewis JJ, Brennan MF. Salvage surgery for patients with recurrent gastrointestinal sarcoma: prognostic factors to guide patient selection. Cancer. 2000;88(1):66-74.

13. van Roggen JF, van Velthuysen MLF, Hogendoorn PCW. The histopathological differential diagnosis of gastrointestinal stromal tumours. J Clin Pathol. 2001;54(2):96-102. 
14. Fletcher CD, Berman JJ, Corless C, Gorstein F, Lasota J, Longley BJ, et al. Diagnosis of gastrointestinal stromal tumors: a consensus approach. Hum Pathol. 2002;33(5):459-65.

15. Pidhorecky I, Cheney RT, Kraybill WG, Gibbs JF. Gastrointestinal stromal tumors: current diagnosis, biologic behavior, and management. Ann Surg Oncol. 2000;7(9):705-12.

16. Wong NA, Young R, Malcomson RD, Nayar AG, Jamieson LA, Save VE, et al. Prognostic indicators for gastrointestinal stromal tumours: a clinicopathological and immunohistochemical study of 108 resected cases of the stomach. Histopathology. 2003;43(2):118-26.

17. Connolly EM, Gaffney E, Reynolds JV. Gastrointestinal stromal tumors. Br J Surg. 2003;90(10):1178-86.

18. D'Amato G, Steinert DM, McAuliffe JC, Trent JC. Update on the biology and therapy of gastrointestinal stromal tumors. Cancer Control. 2005;12(1):44-56.

19. Linhares E, Valadão M. Atualização em GIST. Rev Col Bras Cir. 2006;33(1):51-4.

20. Miettinen M, Sobin LH, Sarmolo-Rikala M. Immunohistochemical spetrum of GISTs at different sites and their differential diagnosis with a reference to CD117 (KIT). Mod Pathol. 2000;13(10):134-42.
21. Aparicio T, Boige V, Sabourin JC, Crenn P, Ducreux M, Le Cesne A, Bonvalot S. Prognostic factors after surgery of primary resectable gastrointestinal stromal tumours. Eur J Surg Oncol. 2004;30(10):1098-103.

22. Joensuu H, Roberts PJ, Sarlomo-Rikala M, Andersson LC, Tervahartiala P, Tuveson D, et al. Effect of tyrosine kinase inhibitor STI571 in a patient with a metastatic gastrointestinal stromal tumor. N Engl J Med. 2001;344(14):1052-6.

23. Englesbe MJ, Pelletier SJ, Diehl KM, Sung RS, Wahl WL, Punch J, Bartlett RH. Transfusions in surgical patients. J Am Coll Surg. 2005;200(2):249-54.

Como citar este artigo:

Valadão M, Lourenço LG, Linhares E, Romano S, Kesley R, Siqueira D. Fatores prognósticos clínicos e anatomopatológicos dos tumores estromais gastrointestinais (GIST) de origem gástrica. Rev Col Bras Cir. [periódico na Internet] 2006 Set-Out;33(5). Disponível em URL: www.scielo.br/rcbc

Endereço para correspondência:

Rua Paissandú, 385/apto.302

Laranjeiras

22210-080 - Rio de Janeiro - RJ

E-mail:marcusvaladao@ig.com.br 